University of Wollongong

Research Online

Australian Institute for Innovative Materials -

Papers

Australian Institute for Innovative Materials

2013

\title{
Structurally homogeneous MgB2 superconducting wires through economical wet mixing process
}

Md Shahriar A. Hossain

University of Wollongong, shahriar@uow.edu.au

Ashkan Motaman

University of Wollongong, am107@uowmail.edu.au

Xun Xu

University of Wollongong, xun@uow.edu.au

Khay Way See

University of Wollongong, kwsee@uow.edu.au

Ozlem Cicek

Ankara University

See next page for additional authors

Follow this and additional works at: https://ro.uow.edu.au/aiimpapers

Part of the Engineering Commons, and the Physical Sciences and Mathematics Commons

Research Online is the open access institutional repository for the University of Wollongong. For further information contact the UOW Library: research-pubs@uow.edu.au 


\title{
Structurally homogeneous MgB2 superconducting wires through economical wet mixing process
}

\author{
Abstract \\ We evaluate the effects of the mixing process between the starting materials on the structural and \\ superconducting properties of carbon-doped MgB2 wires. The critical current density, n-value, amount of \\ $\mathrm{MgO}$, and critical temperature are obviously dependent on the mixing process, while the microstructure, \\ i.e., grain size, is not sensitive. In our study, the wet-mixing process in toluene enables more homogenous \\ mixing between the starting materials, resulting in superior $\mathrm{J}(\mathrm{c})$ and $\mathrm{n}$-value.

\section{Keywords} \\ economical, homogeneous, wires, superconducting, process, mixing, mgb2, wet, structurally \\ Disciplines \\ Engineering | Physical Sciences and Mathematics \\ Publication Details \\ Hossain, M. A., Motaman, A., Xu, X., See, K. Way., Cicek, O., Agil, H., Ertekin, E., Gencer, A., Cheong, K., \\ Maeda, M. \& Dou, S. (2013). Structurally homogeneous MgB2 superconducting wires through economical \\ wet mixing process. Materials Letters, 91 356-358.

\section{Authors} \\ Md Shahriar A. Hossain, Ashkan Motaman, Xun Xu, Khay Way See, Ozlem Cicek, Hasan Agil, Ercan Ertekin, \\ Ali Gencer, Kookchae Cheong, Minoru Maeda, and S X. Dou
}




\title{
Structurally homogeneous $\mathrm{MgB}_{2}$ superconducting wires through economical wet mixing process
}

Md. Shahriar A. Hossain ${ }^{1 *}$, Ashkan Motaman ${ }^{1}$, Xun $\mathrm{Xu}^{1}$, Özlem Çiçek ${ }^{2}$, Hasan Ağıl${ }^{2}$, Ercan Ertekin $^{2}$, Ali Gencer ${ }^{2}$, Kookchae Cheong ${ }^{3}$, Minoru Maeda ${ }^{4}$, Shixue Dou ${ }^{1}$

${ }^{1}$ Institute for Superconducting and Electronic Materials, University of Wollongong, Squires Way, Innovation Campus, North Wollongong, New South Wales 2519, Australia

${ }^{2}$ Ankara University, Faculty of Science, Department of Physics, Tandoğan 06100, Ankara, Turkey

${ }^{3}$ Nano-functional Materials Group, Korea Institute of Materials Science, Changwon, Gyeongnam 642-831, Republic of Korea

${ }^{4}$ Department of Physics, College of Science and Technology, Nihon University, Chiyoda, Tokyo 101-8308, Japan

\begin{abstract}
:
We evaluate the effects of the mixing process between the starting materials on the structural and superconducting properties of carbon-doped $\mathrm{MgB}_{2}$ wires. The critical current density, $n$-value, amount of $\mathrm{MgO}$, and critical temperature are obviously dependent on the mixing process, while the microstructure, i.e., grain size, is not sensitive. In our study, the wet-mixing process in toluene enables more homogenous mixing between the starting materials, resulting in superior $J_{\mathrm{c}}$ and $n$ value.
\end{abstract}

Keywords: magnesium diboride, homogeneity, connectivity, $n$-factor

*Corresponding author: Tel. + 6124221 3384; Fax. 6124221 5731; Email. shahriar@uow.edu.au 
When carbon partially substitutes into boron layers of the $\mathrm{MgB}_{2}$ lattice structure, it is believed that the upper critical field goes up due to impurity scattering [1-5] For this purpose, malic acid $\left(\mathrm{C}_{4} \mathrm{H}_{6} \mathrm{O}_{5}\right)$ is known to be the most promising source, even though it contains oxygen [4, 5]. Even with the oxygen, it was reported that $\mathrm{MgB}_{2}$ wire with malic acid doping showed the best critical current density $\left(J_{\mathrm{c}}\right)$. To achieve this, a wet-mixing process was simultaneously employed, using toluene $\left(\mathrm{C}_{7} \mathrm{H}_{8}\right)$ as the mixing medium to eliminate oxygen [4-6]. The conjectured role of the wetmixing process has been not proved, however. Herein, we have selected tartaric acid $\left(\mathrm{C}_{4} \mathrm{H}_{6} \mathrm{O}_{6}\right)$ as the carbon source, although it has slightly higher oxygen content compared to malic acid. According to some early studies [7], hydrocarbons such as toluene and benzene have been in only limited use as carbon dopants because their high volatility limits the carbon substitution level. It might be expected that the use of toluene as a mixing medium would enable more homogenous mixing between the starting materials. This study, therefore, is an investigation of the effects of a wet-mixing process on the structural and superconducting properties of powder-in-tube $\mathrm{MgB}_{2}$ wires. We establish a strong correlation between the mixing process and the $J_{\mathrm{c}}, n$-value, amount of $\mathrm{MgO}$, and critical temperature $\left(T_{\mathrm{c}}\right)$.

$\mathrm{MgB}_{2} / \mathrm{Fe}$ monofilament wires with $10 \mathrm{wt} \%$ tartaric acid additive were prepared by dry- and wetmixing processes. The fabrication process for the different powders has been published elsewhere [4-6]. Tartaric acid (99\%, Aldrich), magnesium (99.8\%, 325 mesh), and boron (99\%, 2-30 nm) were used as starting materials. The different powder mixtures were packed into iron $(\mathrm{Fe})$ tubes 8 $\mathrm{mm}$ in outer diameter and $5 \mathrm{~mm}$ in inner diameter. The composites were drawn into a wire $1.1 \mathrm{~mm}$ in diameter. The wire was then sintered at $900^{\circ} \mathrm{C}$ for 30 min under argon atmosphere. Scanning electron microscopy (SEM) was employed to observe the morphology. X-ray diffraction measurements were performed to identify the phase composition and extract structural parameters from Rietveld refinement. Transport critical current up to 250 A was measured by using the 
standard four-probe method with a criterion of $1 \mu \mathrm{Vcm}^{-1}$. Resistivity was measured with the standard AC four-probe method.

Transport $J_{\mathrm{c}}$ curves at $4.2 \mathrm{~K}$ and $20 \mathrm{~K}$ for samples produced using different mixing processes such as wet and dry mixing are presented in Figure 1(a). The $J_{\mathrm{c}}$ of the wet-mixed wire is as high as $10,000 \mathrm{~A} / \mathrm{cm}^{2}$ at $9.6 \mathrm{~T}$ and $4.2 \mathrm{~K}$. The corresponding value is $8.7 \mathrm{~T}$ for wire treated through drymixing. This indicates that the wet mixing is much more effective with a carbohydrate dopant, i.e., tartaric acid. Interestingly, the critical current densities at $20 \mathrm{~K}$ showed the same trend. In order to find other clues, we evaluated the normal state resistivity of the three wires, as summarized in Table I. It is generally argued that the residual resistivity, $\rho_{40 \mathrm{~K}}$, is related to the intragrain impurity scattering, whereas the difference between the residual resistivity and the room temperature resistivity, $\Delta \rho=\rho_{300 \mathrm{~K}}-\rho_{40 \mathrm{~K}}$, is affected by the intergrain connectivity [8]. From the resistivity measurements, grain connectivity was further investigated. First, the residual resistivity ratio (RRR), simply defined as the ratio of the resistivity at $300 \mathrm{~K}$ to the resistivity at $40 \mathrm{~K}$, was $1.93,1.76$, and 1.67 for the un-doped, wet-mixed, and dry-mixed wires, respectively. Samples with relatively high values of RRR are known to be high quality [9]. Second, the active cross-sectional area fraction $\left(A_{F}\right)$ is defined by $[8]$

$\mathrm{A}_{\mathrm{F}}=\Delta \rho_{\text {ideal }} /\left(\rho_{300 \mathrm{~K}}-\rho_{40 \mathrm{~K}}\right)$

where $\rho_{40 \mathrm{~K}}$ and $\rho_{300 \mathrm{~K}}$ are the resistivity measured at $40 \mathrm{~K}$ and $300 \mathrm{~K}$, respectively. $\Delta \rho_{\text {ideal }}$ is the resistivity difference between $40 \mathrm{~K}$ and $300 \mathrm{~K}$ for an ideal sample, and the value of $7.3 \mu \Omega \mathrm{cm}$ is typically used [10]. The area fractions for un-doped, wet-mixed, and dry-mixed wires were 0.214 , 0.128 , and 0.117 , respectively. This result also supports the better efficiency of the wet-mixing process from the viewpoint of grain connectivity. As was noted, it was proved that the impurity scattering between the $\sigma$ and $\pi$ bands can affect the $J_{c}$ of $\mathrm{MgB}_{2}$ wire, as can be seen in Figure 1(a) and Table 1. The field dependence of the $n$-value for the three kinds of $\mathrm{MgB}_{2}$ wire is shown in Figure 1(b). In general, the $n$-value of a conductor/wire plays an important role in predicting (i) the 
decay property of joints and (ii) the electrical dissipation [11, 12]. Here, $n$-values were determined from the slope in the plot of $\log E$ versus $\log J$ in the $E$ range from 0.1 to $10 \mu \mathrm{V} / \mathrm{cm}^{-1}$, based on the power law, $E_{\mathrm{c}}=E\left(V / V_{\mathrm{c}}\right)^{n}$, where the subscripted c refers to the critical values. From the Figure, the $n$-value behaviour shows the same trend as the $J_{\mathrm{c}}$. Interestingly enough, the wet-mixed $\mathrm{MgB}_{2}$ wire had higher $n$-values compared to the un-doped and dry-mixed wires. The higher $n$-value means that improved homogeneity and grain connectivity may be obtained in the superconducting core through powder processing by the wet-mixing process. This result is of importance from the viewpoint of mass production.

The power-law relationship between the critical current densities and the $n$-values of wires evaluated over all temperature ranges is shown in Figure 2. In general, the power-law relationship ( $m$ ) between the critical curent density $\left(J_{\mathrm{c}}\right)$ and the $n$-value, $n \square J_{\mathrm{c}}{ }^{m}$, represents a critical index, which is strongly dependent on the mixing process, as observed in our study. The index $m$ values were estimated to be $0.563,0.539$, and 0.509 , respectively, for the wet-mixed, dry-mixed, and undoped wires. We now conclude that a larger $J_{\mathrm{c}}$ leads to a higher $n$-value. As was noted, the resistance component due to the $n$-value can be effectively reduced when a conductor with high $n$ value is used as a magnet material.

A natural question is then what is the main reason for the enhancement of $J_{\mathrm{c}}$ through the wet-mixing process. In order to explore this question, we evaluated the structural parameters through Rietveld refinement, as presented in Figure 3(a). Here, the weight fraction of $\mathrm{MgO}$ for the wire treated by dry mixing increased with increasing amounts of tartartic acid, from 5 to $30 \mathrm{wt} \%$. This is due to the oxygen content from tartaric acid. Specifically, the oxygen in the tartaric acid reacts with residual magnesium to form the $\mathrm{MgO}$. In contrast, the amount of $\mathrm{MgO}$ for the wire treated by wet mixing is independent of the tartaric acid content. This means that the wet-mixing helps to eliminate residual oxygen during powder processing. $T_{\mathrm{c}}$ is slightly decreased, however, for the wire treated by wet mixing (Figure 3(b)). This can be related to the amount of carbon substitution. It is believed that more carbon substitutes into the boron layers of the $\mathrm{MgB}_{2}$ lattice structure, resulting in reduction of 
the $T_{\mathrm{c}}$. The formation of $\mathrm{MgO}$, however, is responsible for the larger drop in the $T_{\mathrm{c}}$ in the case of dry mixing rather than carbon substitution. This result obviously indicates that the dry mixing leads to inhomogeneity due to the large fraction of $\mathrm{MgO}$.

Figure 4 shows scanning electron microscope (SEM) images of wires treated through wet and dry mixing. The morphologies are likely to be the same. In particular, the grain size seems to remain the same through the different mixing processes, but the dry mixed samples look agglomerated. Wet mixed wires seem to have more homogeneous and connected grains than dry mixed ones. This means that the two wires are subject to a similar grain boundary pinning strength.

In summary, we have evaluated the effects of the mixing process between the starting materials, either dry mixing or wet mixing in toluene, on the structural and superconducting properties of carbon-doped $\mathrm{MgB}_{2}$ wires. The $J_{\mathrm{c}}, n$-value, amount of $\mathrm{MgO}$, and $T_{\mathrm{c}}$ were all dependent on the mixing process, while the microstructure remained the same. The $J_{\mathrm{c}}$ and $n$-value at $4.2 \mathrm{~K}$ and $10 \mathrm{~T}$ were estimated to be $8000 \mathrm{~A} / \mathrm{cm}^{2}$ and 30, respectively, with the liquid mixing process. This can be related to less $\mathrm{MgO}$ formation in the matrix. We therefore conclude that the wet-mixing process in toluene enables more homogenous mixing between the starting materials with tartaric acid as the carbon source.

Acknowledgements We gratefully acknowledge Dr. Jung Ho Kim, for helpful discussions. This work was supported by the Australian Research Council (Project number: LP120100173). 


\section{REFERENCES}

1) H. Kumakura, H. Kitaguchi, A. Matsumoto, and H. Hatakeyama: Appl. Phys. Lett. 84 (2004) 3669.

2) S. X. Dou, S. Soltanian, J. Horvat, X. L. Wang, S. H. Zhou, M. Ionescu, H. K. Liu, P. Munroe, and M. Tomsic: Appl. Phys. Lett. 81 (2002) 3419.

3) S. X. Dou, O. Shcherbakova, W. K. Yeoh, J. H. Kim, S. Soltanian, X. L. Wang, C. Senatore, R. Flukiger, M. Dhalle, O. Husnjak, and E. Babic: Phys. Rev. Lett. 98 (2007) 097002.

4) J. H. Kim, S. Zhou, M. S. A. Hossain, A. V. Pan, and S. X. Dou: Appl. Phys. Lett. 89 (2006) 142505.

5) M. S. A. Hossain, J. H. Kim, X. Xu, X. L. Wang, M. Rindfleisch, M. Tomsic, M. D. Sumption, E. W. Collings, and S. X. Dou: Supercond. Sci. Technol. 20 (2007) L51-4.

6) M. S. A. Hossain, J. H. Kim, X. L. Wang, X. Xu, G. Peleckis, and S. X. Dou: Supercond. Sci. Technol. 20, (2007) 112116.

7) H. Yamada, M. Hirakawa, M. Kumakura, and H. Kitaguchi: Supercond. Sci. Technol. 19 (2006) 175.

8) J. Rowell et al., Supercond. Sci. Technol. 16 (2003) R17-27.

9) T. Matsushita, M. Kiuchi, A. Yamamoto, J. I. Shimoyama, and K. Kishio: Supercond. Sci. Technol. 21 (2008) 015008.

10) M. Eisterer et al.: Supercond. Sci. Technol. 20 (2007) R47-73.

11) J. H. Kim, S. X. Dou, A. Matsumoto, S. Choi, T. Kiyoshi, and H. Kumakura: Physica C 470 (2010) 1207-1210.

12) M. Takahashi, K. Tanaka, M. Okada, H. Kitaguchi, and H. Kumakura: IEEE Trans. Appl. Supercond. 16 (2006) 1431.

\section{Figure captions:}

Fig. 1: Magnetic field dependence of (a) $J_{\mathrm{c}}$ and (b) $n$-value for wires fabricated through wet and dry mixing. The corresponding values for un-doped wires are also included for reference.

Fig. 2: Correlation between $J_{\mathrm{c}}$ and $n$-value for wires fabricated through wet and dry mixing. The corresponding values for un-doped wires are also included for reference.

Fig. 3: (a) Weight fraction of $\mathrm{MgO}$ of wires fabricated through wet and dry mixing with different amounts of tartaric acid, and (b) $T_{\mathrm{c}}$ dependence on the amount of tartaric acid.

Fig. 4: SEM images of wires fabricated through (a) dry and (b) wet mixing.

Table I: The measured resistivity values, residual resistivity ratio, and active cross-sectional area fraction for wires fabricated through wet and dry mixing.

\begin{tabular}{cccccc}
\hline \hline Samples & $\begin{array}{c}\boldsymbol{\rho}_{\mathbf{3 0 0} \mathbf{K}} \\
(\boldsymbol{\mu} \boldsymbol{\Omega} \mathbf{c m})\end{array}$ & $\begin{array}{c}\boldsymbol{\rho}_{\mathbf{4 0 ~ K}} \\
(\boldsymbol{\mu} \boldsymbol{\Omega} \mathbf{c m})\end{array}$ & $\boldsymbol{\Delta \rho}(\boldsymbol{\mu} \mathbf{\Omega c m})$ & $\mathbf{R R R}$ & $\mathbf{A}_{\mathbf{F}}$ \\
\hline \hline Undoped $\mathrm{MgB}_{2}$ & 70.55 & 36.45 & 34.10 & 1.93 & 0.214 \\
$\mathrm{MgB}_{2}+10 \%$ tart & 131.56 & 74.67 & 56.89 & 1.76 & 0.128 \\
$\begin{array}{c}\text { (wet) } \\
\mathrm{MgB}_{2}+10 \% \text { tart } \\
\text { (dry) }\end{array}$ & 154.85 & 92.56 & 62.29 & 1.67 & 0.117 \\
\hline \hline
\end{tabular}



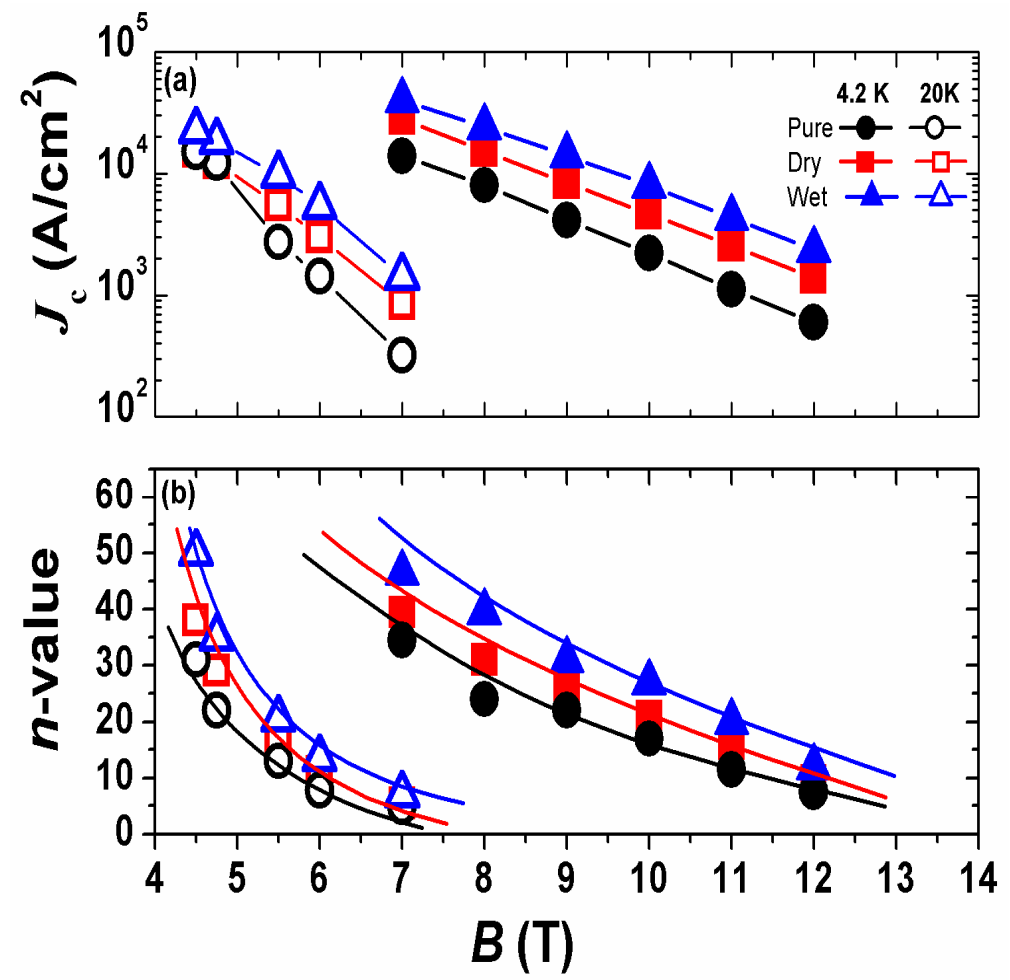

Fig. 1

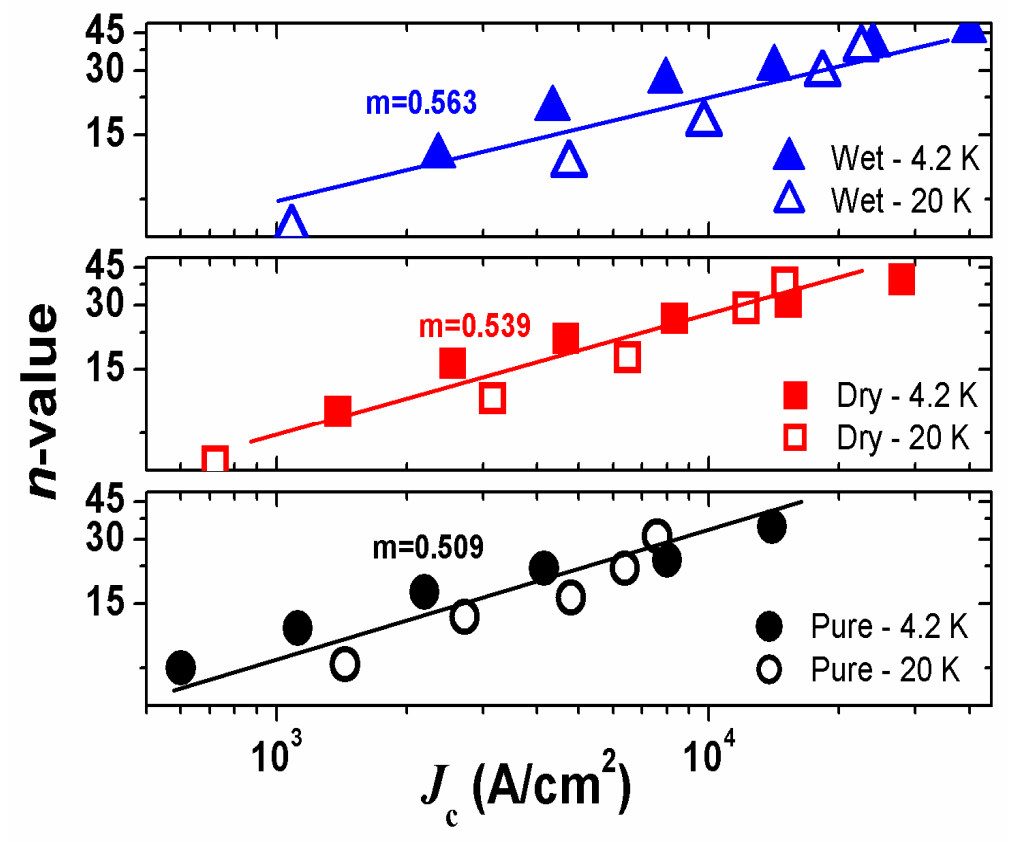

Fig. 2 


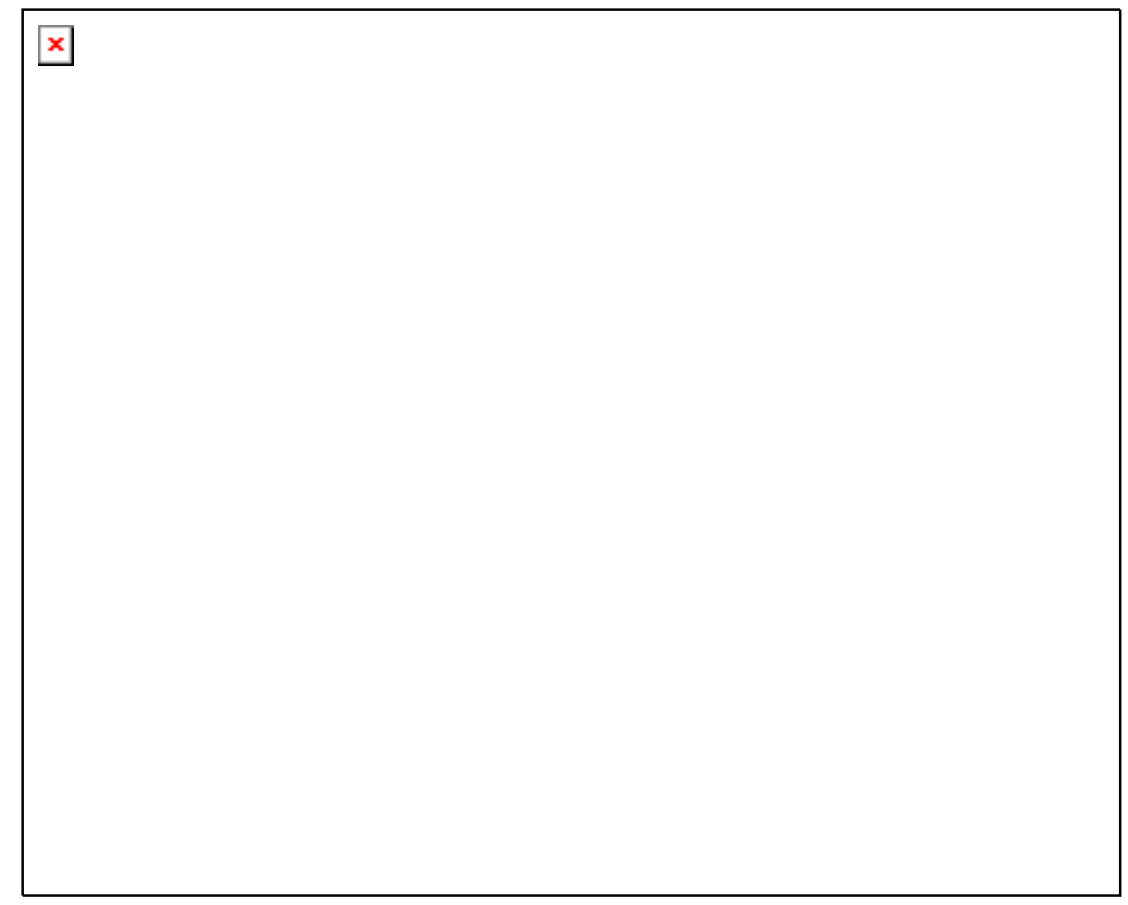

Fig. 3
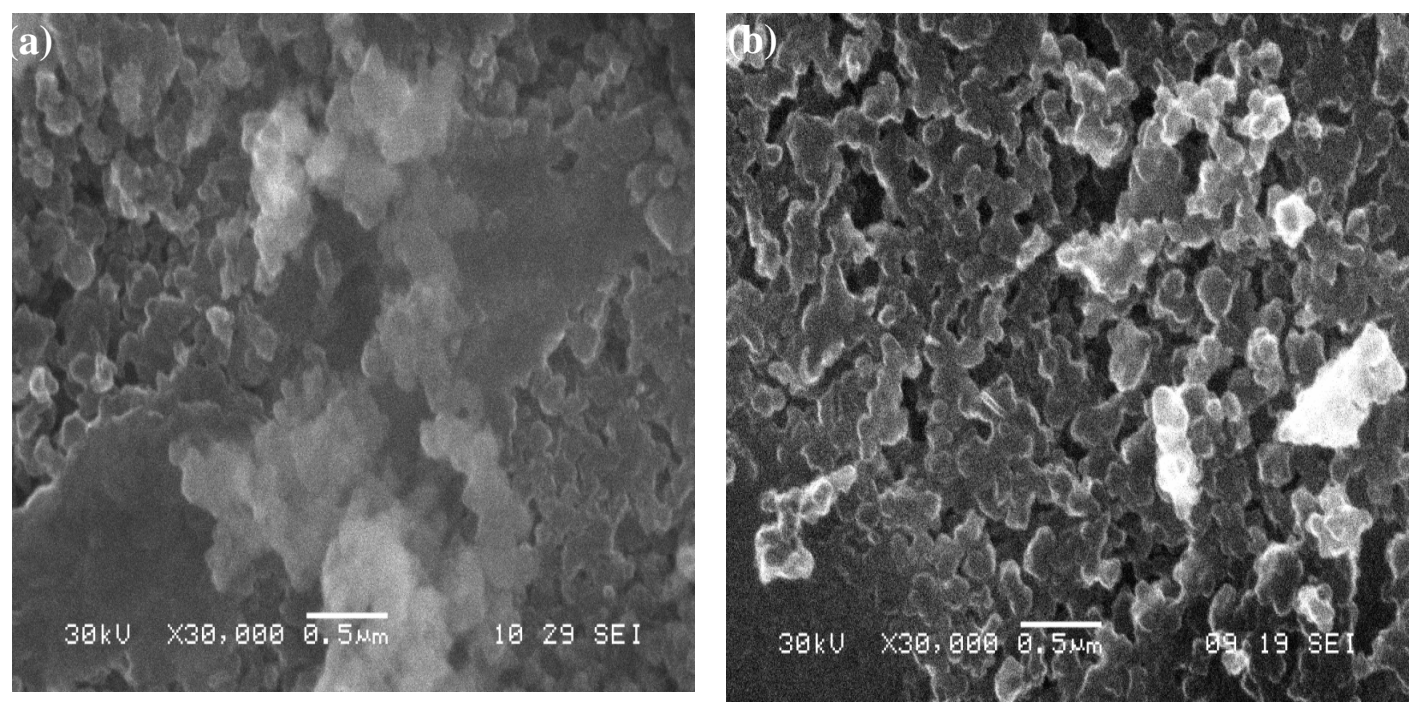

Fig. 4 